\title{
Structure / Property (Constitutive and Dynamic Strength / Damage) Behavior of Additively Manufactured Tantalum
}

\author{
George.T. Gray III ${ }^{1 *}$, Veronica Livescu ${ }^{1}$, Cameron Knapp, David R. Jones, Saryu Fensin, Shuh-Rong Chen, Carl \\ M. Cady, Carl P. Trujillo, and Daniel Martinez ${ }^{1}$
}

Los Alamos National Laboratory, Los Alamos, New Mexico, 87545, USA

\begin{abstract}
For Certification and qualification of an engineering component generally involves meeting engineering and physics requirements tied to its functional requirements. In this paper, the results of a study quantifying the microstructure, mechanical behavior, and the dynamic damage evolution of Tantalum (Ta) fabricated using an EOS laser-powder-bed machine are presented. The microstructure and quasi-static mechanical behavior of the AM-Ta is detailed and compared / contrasted to wrought Ta. The dynamic damage evolution and failure response of the AM-Ta material, as well as wrought Ta, was probed using flyer-plate impact driven spallation experiments. The differences in the spallation response between the AM and wrought Ta were measured using in-situ velocimetry as well as post-mortem quantification of damage in "soft-recovered" samples. The damage evolution of the AM and wrought Ta were characterized using both optical metallography and electron-backscatter diffraction.
\end{abstract}

\section{Introduction}

Additive manufacturing (AM) is a rapidly evolving area of materials science and technology driving rapid changes in prototyping, freeform, and net-shape manufacturing. Several recent reviews have summarized the state-of-theart of metal additive technologies [1-3]. The AM of metallic components offers numerous opportunities for improved efficiencies in energy needs, cost per part, and time savings during manufacturing $[2,4,5]$. Alternately, AM manufacturing of metallic components must be carefully viewed within the reality of the high expense of AM machines, high feedstock costs, limited build volumes, slow build rates, and complex and largely unproven certification / qualification procedures / requirements for high-performance components [2-3]. Finally, a lack of quantified and certified processingstructure-properties-performance (PSPP) data, due to published research lacking documentation of the specifics of: 1) feedstock pedigree including source and chemistry, 2) specific AM machine build settings, 3) quantified part / build thermal histories, 4) detailed part-orientationmechanical testing property correlations, and 5) 3D quantified microstructure and defect distribution and statistics, all hamper efforts to develop a robust roadmap to certifying and qualifying AM metals and alloys.

Resistance to damage during impact / shock-loading environments is of relevance to utilization of AM materials in high-rate manufacturing (forging or machining), crashworthiness (aerospace and automotive), and defense applications [6]. However, while there is a growing number of detailed structure-property studies of AM materials subjected to quasi-static and/or fatigue loading stress states, there remains to date few studies systematically quantifying their behavior at higher strain rates, shock loading, and/or when subjected to dynamic tensile 1D spallation loading [7-9]. Further, AM manufacturing of refractory pure metals and alloys has to date received little attention in contrast to alloys germane to aerospace and other industrial applications. The purpose of this paper is to report shock-loading-induced dynamic fracture (spallation) properties as a function of build orientation of Ta produced by laser-powder-bed additive manufacturing in comparison to annealed wrought Ta.

\section{Experimental Procedures}

\subsection{Material - Processing}

This study involved production, characterization and testing of a plate of Tantalum (hereafter Ta) produced by laser powder-bed additive manufacturing and contrasted to wrought $\mathrm{Ta}$. The wrought Ta was procured in 10.16 $\mathrm{mm}$-thick plate form and displayed a nominally equiaxed $45 \mu \mathrm{m}$ grain size. The crystallographic texture of the wrought Ta was investigated using X-ray diffraction to be nominally 4 times random $<111>$ fiber texture. The highpurity Ta powder used for the additive process was procured from Tekna Advanced Materials, Inc. (Sherbrooke, QC, Canada). An EOS M280 laser-powderbed machine was used to fabricate the tantalum blocks. The 5-45 $\mu \mathrm{m}$ diameter spherical tantalum powder was plasma spherodized and treated in a fluidized bed to remove any satellites to ensure good flowability,

\footnotetext{
* Corresponding author: rusty@lanl.gov
} 
conducive to good spreading in a powder bed format. Elemental Ta has an elevated enthalpy of melting, $10.67 \mathrm{~J} / \mathrm{mm}^{3}$, requiring the maximum volumetric energy density that can be realistically obtained from a 400 Watt variant of the EOS M280. The settings were chosen to obtain a balance between maximum volumetric energy density and maintaining a stable molten pool that avoided surface tension driven solidification. Surface tension driven solidification would have resulted in an unacceptable surface roughness that would have led to machine jams, recoater blade damage, and elevated porosity. The AM plates were built vertically on a subsize Ta insert that was fixed to a larger stainless steel build plate. The build plate assembly was held at $200{ }^{\circ} \mathrm{C}$ during fabrication. Fabrication of a single $50.8 \mathrm{~mm} \times 12.5$ $\mathrm{mm}$ x $101.6 \mathrm{~mm}$ AM-Ta-plate required 85 hours of machine build time.

Sample preparation of the wrought and AM-Ta samples consisted of grinding on $\mathrm{SiC}$ paper with increasingly fine grit, followed by mechanical polishing with $0.3 \mu \mathrm{m}$ alpha alumina slurry and then a mixture of 5:1 by volume of $0.04 \mu \mathrm{m}$ colloidal silica and hydrogen peroxide. Electron Backscatter Diffraction (EBSD) [1011] was employed to investigate the cross sections of the samples following preparation for optical metallography. In order to reveal the microstructure and in preparation for EBSD analysis, the samples were lightly etched for 5 seconds in a solution of $10 \mathrm{ml}$ nitric acid, $10 \mathrm{ml}$ hydrofluoric acid, and $30 \mathrm{ml}$ sulfuric acid. The microstructure and the orientation of the AM-Ta samples studied are presented in Figure 1.

In contrast to the wrought Ta, the AM-Ta exhibits an elongated grain structure akin to a "stack of straws" morphology with the elongated grains parallel to the build direction. Accordingly, the microstructure from the top of the build (termed the in-plane (IP) direction) shows nominally small equiaxed grains, while when viewed from the face of the AM-Ta plate (termed the thruthickness (TT) direction) elongated grains are observed. In addition, detailed metallographic examination of the cross-section of the AM-Ta plate revealed that while the majority of the AM-Ta build plate was fully dense, the upper $\sim 25 \%$ of the plate displayed small solidification flaws / planar lack of fusion flaws resulting in a lack of full density in the upper portion of this plate. The chemistry (in wt.\% ppm) of the as-built AM-Ta and the wrought Ta is presented in Table I.

The differences in chemistry between the wrought and AM-Ta are significant and thought to reflect: 1) interstitial pick-up during AM build both from the powder itself as well as the inert nitrogen build environment, and 2) regardless of the special care in cleaning the EOS machine between builds, the elemental analysis in the AM-Ta strongly suggests the usage of the EOS machine to previously built 304L SS, Ti-6Al-4V, and AlloSiMg components has led to these elements being incorporated into the AM-Ta build.

To probe the effect of these build flaws, as well as the effect of the morphological anisotropy in the elongated grain structure, spall samples were sectioned from: 1) the AM-Ta plate in both the orthotropic directions from the fully dense portion of the plate, labeled "solid", and 2) a single sample from the in-plane orientation from the region possessing the build flaws, termed "porous-inplane" to directly compare with the fully-dense "solid-inplane" sample.

Table 1. Chemistry in Ta investigated.

\begin{tabular}{|l|l|l|l|l|l|l|}
\hline & $\mathrm{O}$ & $\mathrm{N}$ & $\mathrm{Al}$ & $\mathrm{Si}$ & $\mathrm{Ti}$ & $\mathrm{V}$ \\
\hline Wrought & 41 & 1 & $<.005$ & .32 & $<.001$ & $<.005$ \\
\hline AM-Ta & 410 & 83 & 11 & 4.4 & 91 & 4.6 \\
\hline & & & & & & \\
\hline & $\mathrm{Cr}$ & $\mathrm{Fe}$ & $\mathrm{Ni}$ & $\mathrm{Nb}$ & $\mathrm{W}$ & $\mathrm{Ta}$ \\
\hline Wrought & $<.001$ & .008 & $<.005$ & 61 & 43 & bulk \\
\hline AM-Ta & 100 & 220 & 33 & 21 & 45 & bulk \\
\hline
\end{tabular}
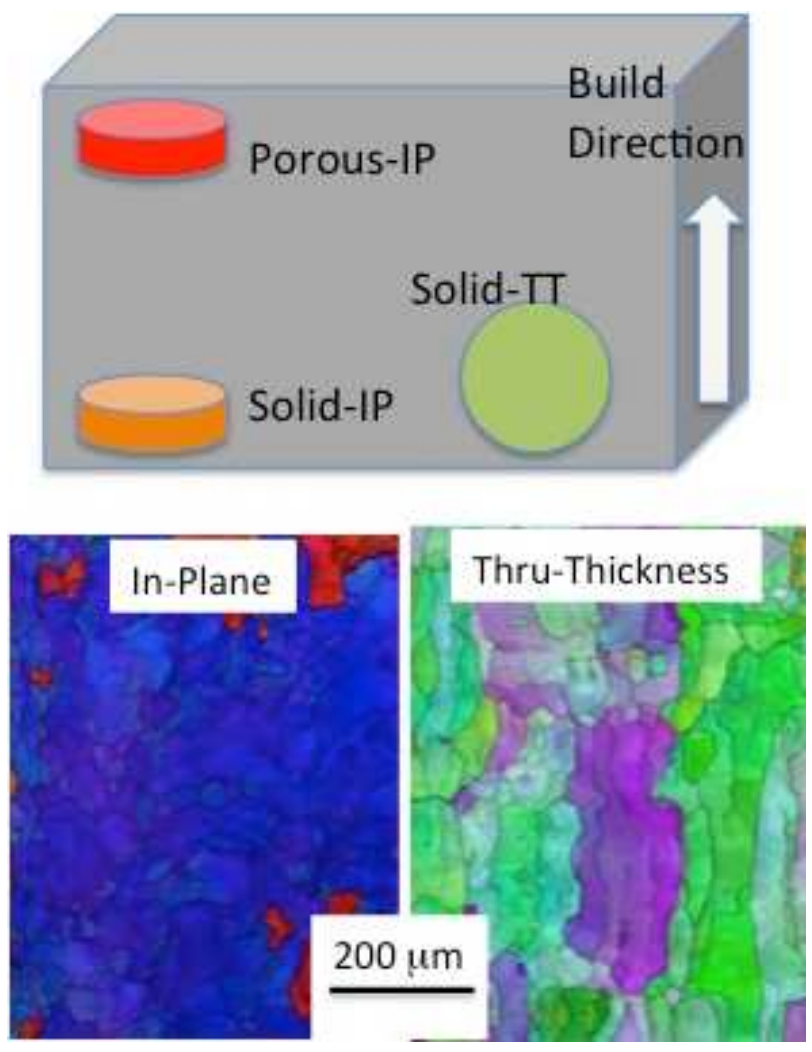

Fig. 1. Schematic of spall sample locations for the In-plane (IP) and Thru-Thickness (TT) orientations from the AM-Ta plate, and optical metallography of the columnar microstructure of the AM-Ta build as a function of direction.

\subsection{Constitutive / Spallation Characterization}

Spallation experiments were nominally conducted as described previously [12]. Three AM-Ta samples sectioned from the AM-Ta plate as denoted in Figure 1 as well as one wrought sample were simultaneously subjected to spall loading in a single assembly to assure identical loading conditions. Impactors of wrought Ta were accelerated using a $80 \mathrm{~mm}$ bore gas gun at a velocity of $208 \mathrm{~m} / \mathrm{s}$ and impacted onto the four Ta-target assembly. All impactors were nominally $2.5 \mathrm{~mm}$ thick. Each target consisted of three separate components: a target plate, the four Ta samples of different diameters, and a momentum ring for each sample. Targets were 5 $\mathrm{mm}$ thick and designed specifically such that recovery of 
the shocked samples was facilitated for post-mortem examination. The samples were press-fit into the momentum rings and the rings press-fit into the target plate to ensure intimate contact of all components initially. For the four-sample target, samples were machined as right circular cylinders 8 to $12 \mathrm{~mm}$ in diameter from the wrought and the as-built AM-Ta material as shown in Figure 2. In the experiment, the impactor thickness was one-half the sample thickness to cause tensile damage to occur in the center of the samples. The single wrought and three AM-Ta samples were placed in the target ring such that the shortest distance between the four sample edges to each other and to the edge of the target plate were equal. Each sample was surrounded by momentum trapping rings made from wrought Ta with an inner diameter (ID) $0.1 \mathrm{~mm}$ less than the outer diameter (OD) of the sample. The assembled targets were lapped flat and parallel to within $5 \mu \mathrm{m}$.

Free surface velocities were measured in each experiment using Photon Doppler Velocimetry (PDV) [13]. Collimated probes provided by AC Photonics (Part \#: 1CL15P020LC-C01) were positioned to collect velocimetry data from the center of each sample and connected to a LANL-built 4-channel PDV system. A single PZT trigger pin (Dynasen, Inc.) was also positioned next to each target plate with a typical standoff distance of a few millimeters to provide a trigger to the digitizer used to collect the PDV data. The Ta specimens following spallation loading were cross-sectioned and prepared for optical metallography [14].

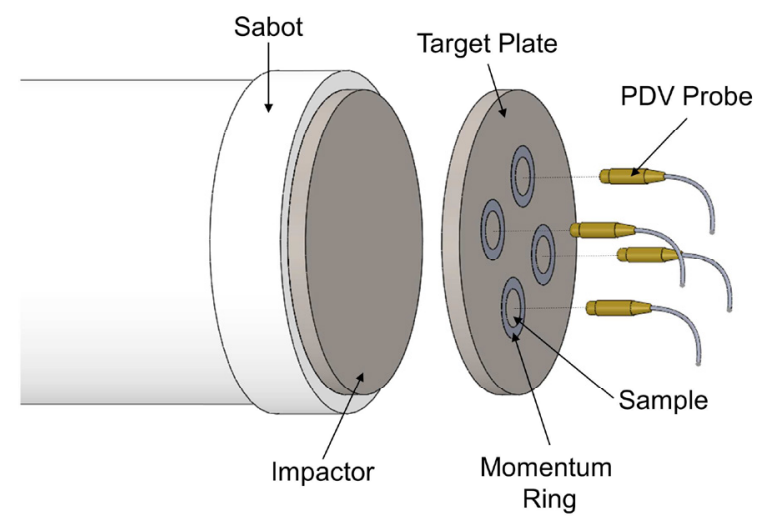

Fig. 2. Experimental configuration used to produce incipient damage or complete spall in four samples simultaneously. Wrought Ta impactors were accelerated in a $80 \mathrm{~mm}$ gas gun and impacted onto the target. PDV probes were placed over the center of each sample to provide free-surface velocimetry data from each sample in each experiment.

\section{Results and Discussion}

\subsection{Constitutive Response}

The quasi-static compressive constitutive response of the AM and wrought Ta materials is shown in Fig. 3. The asbuilt AM material exhibits twice the yield strength of the annealed wrought plate and exhibits anisotropy in its mechanical behavior unlike the wrought Ta whose TT and IP directions show essentially identical mechanical behavior. This anisotropy in the AM-Ta leads to the compression samples for the AM-IP directions evolving into an elliptical shape following compression to 0.30 . In addition, following yielding, the AM-TT displays a similar work-hardening to the wrought Ta while the AMIP sample displays a higher rate of work hardening than the other three curves in Figure 3. This higher rate of hardening for the AM-IP direction is however seen to display a maximum and thereafter decreases after a true strain of $\sim 0.20$ and decreases so as to attain approximately the same flow stress as the AM-TT sample by a true strain of $\sim 0.30$. To evaluate if the higher flow stress levels in the AM-as-built material was indicative of a higher starting dislocation density or from other sources, strain rate jump tests were conducted at $298 \mathrm{~K}$.

The magnitude of flow stress increase with the strain rate jumps was seen to be slightly less than the wrought $\mathrm{Ta}$ as presented in Figure 4. The somewhat lower flow stress increase with increasing strain rate in the rate jump response suggests the higher starting yield and flow stress displayed by the AM-as-built Ta is due to an intrinsic barrier component to the strength[15] such as: 1) the smaller grain size in the AM-Ta, 2) the increased interstitial content $(\mathrm{O}$ and $\mathrm{N})$, and 3 ) the increased $\mathrm{Fe}, \mathrm{Ti}$, $\mathrm{Ni}, \mathrm{Cr}$, and $\mathrm{V}$ solute levels in the AM-Ta and not related to either a high starting dislocation defect population or residual stresses.

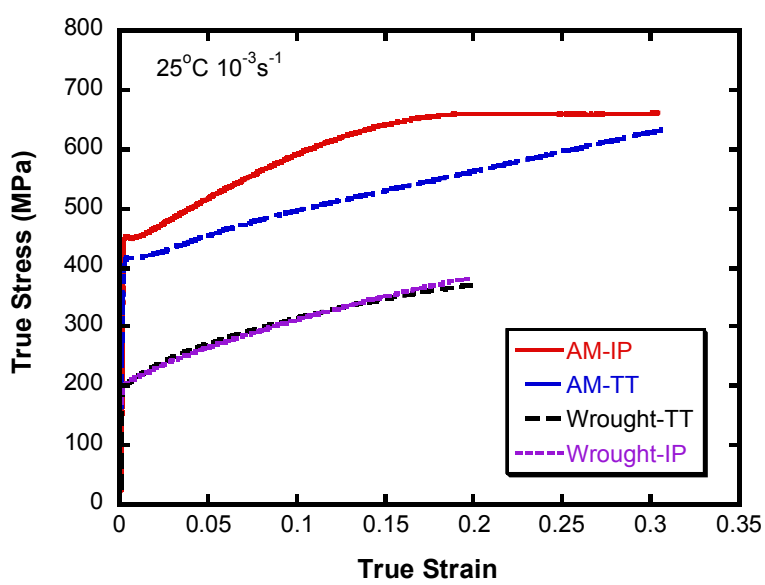

Fig. 3. Compressive True Stress-True Strain of Ta in the AMas-built condition, and wrought Ta along orthogonal directions.

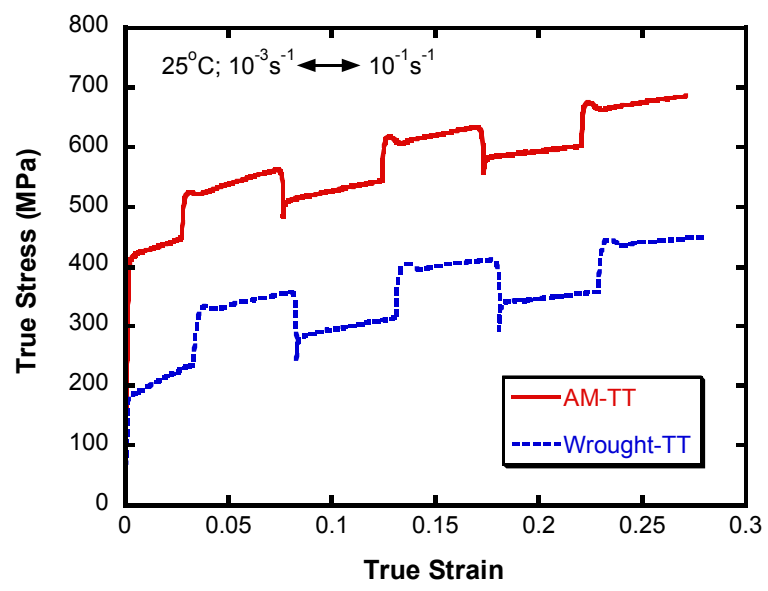

Fig. 4. Strain-rate jump compressive true stress-true strain of the wrought and AM-Ta. 


\subsection{Velocimetry}

Figure 5 shows the PDV wave profiles for the wrought and three AM-Ta samples; both a plot showing the full profiles and an expanded view of the Hugoniot-Elastic Limit (HEL) loading region in Figure 6. The HEL's and the depth of the "pull-back" signals, reflecting the spall strengths of the four materials, each illustrate substantial differences between the dynamic response of the wrought and AM-Ta samples as well as a pronounced influence of loading direction relative to the AM build direction. Quantitatively, the spall strengths, calculated from the perspective of the simple spall model [16], and the HEL's of the wrought and three AM-Ta samples are presented in Table II. From the loading portions of the profiles it is readily observed that all three AM-Ta samples exhibit higher HEL's than the wrought Ta.

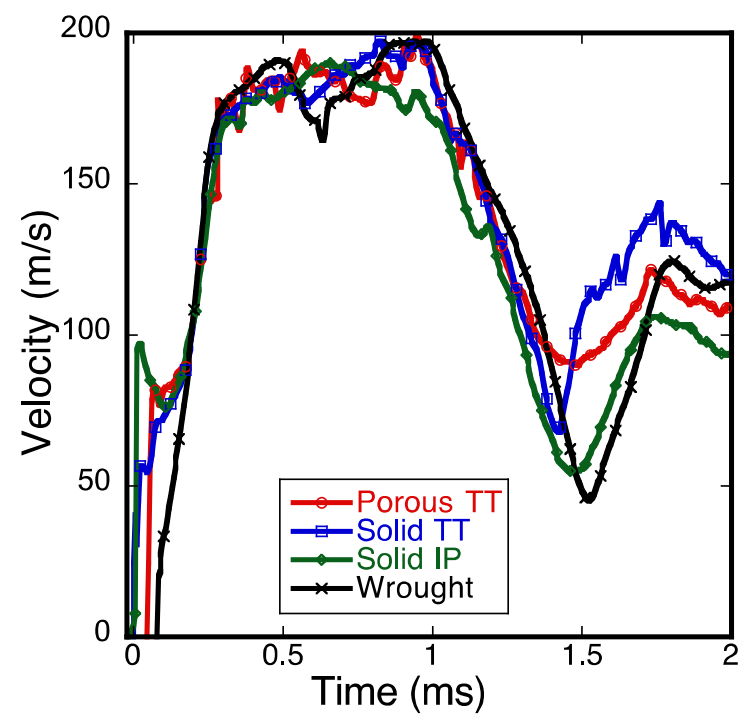

Fig. 5. PDV wave profiles (particle velocity versus time plot) for the wrought and $3 \mathrm{AM}$-Ta materials impacted at $257 \mathrm{~m} / \mathrm{sec}$.

Similarly, the spall strengths show significant differences with the wrought Ta displaying the highest spall strength, followed by the AM-Ta in the TT and IP directions displaying very similar spall strength. The fully-dense TT and porous TT samples, where the porous region contains build flaws, displaying the lowest resistance to spallation.

The higher HEL's for the AM-Ta in contrast to the wrought is consistent with the substantially refined microstructural scale of the AM-Ta and increased solute content leading to higher yield strengths[9]. In contrast, but as previously documented in many materials, the inverse is seen in the spall strengths for the wrought versus AM-Ta samples where the annealed wrought Ta displays a slightly higher resistance to damage evolution than the higher yield strength AM-Ta samples. The difference in the magnitude of the "pull-back" signals for the three materials and the calculated spall strengths is presented for the three AM-Ta in Table II.

The spall strength $\left(\sigma_{\text {spall }}\right)$ was calculated using the relationship for a material that exhibits an elasto-plastic behavior [17]:

$$
\sigma_{\text {spall }} \equiv \rho_{0} C_{L} \Delta F S V\left(1+\frac{C_{L}}{C_{o}}\right)^{-1}
$$

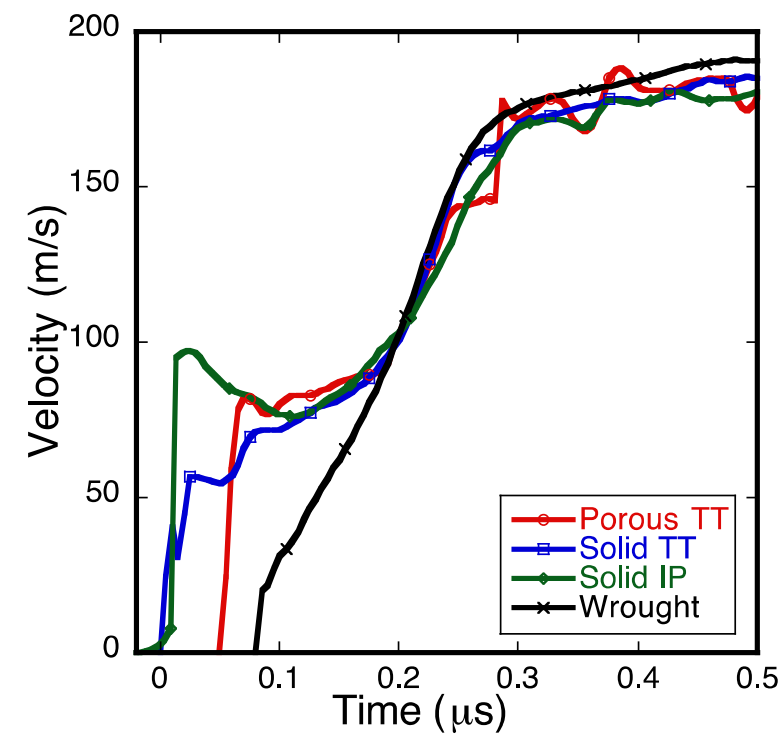

Fig. 6. Expanded view of HEL region in Figure 5 (PDV wave profiles (particle velocity versus time plot) for the wrought and 3 AM-Ta.

where $\mathrm{C}_{\mathrm{L}}=4130 \mathrm{~m} / \mathrm{s}$ is the longitudinal sound speed, $\mathrm{C}_{0}$ is $3430 \mathrm{~m} / \mathrm{s}$, and $\triangle \mathrm{FSV}$ is the difference in the free surface velocity from the peak state to the minima.

Table 2. Calculated Spall Strengths and Hugoniot Elastic Limits (HEL's) for the Four Ta samples.

\begin{tabular}{|c|c|c|}
\hline Sample & $\begin{array}{c}\text { HEL } \\
\text { (GPa) }\end{array}$ & $\begin{array}{c}\text { Spall Strength } \\
\text { (uncorrected) - GPa }\end{array}$ \\
\hline Wrought & 1.0 & 4.1 \\
\hline Porous - TT & 2.8 & 3.1 \\
\hline Solid - IP & 3.3 & 3.8 \\
\hline Solid - TT & 1.9 & 3.7 \\
\hline
\end{tabular}

\subsection{Post-Mortem Metallurgical Analysis}

The four samples from the spallation experiment were cross-sectioned across the diameter of the sample, then ground and polished and thereafter studied as described in section 2.1. Optical metallography of the cross-sections for the wrought $\mathrm{Ta}$ and the two solid AM-Ta samples is presented in Figure 7. Classic nearly-spherical void nucleation and growth is seen in the wrought $\mathrm{Ta}$ as observed and documented previously for annealed highpurity wrought $\mathrm{Ta}$ [18]. Void nucleation was observed to occur principally at grain boundaries and/or grain triple points. This ductile damage evolution mechanism is consistent with the established high spall strength in annealed wrought $\mathrm{Ta}$. 

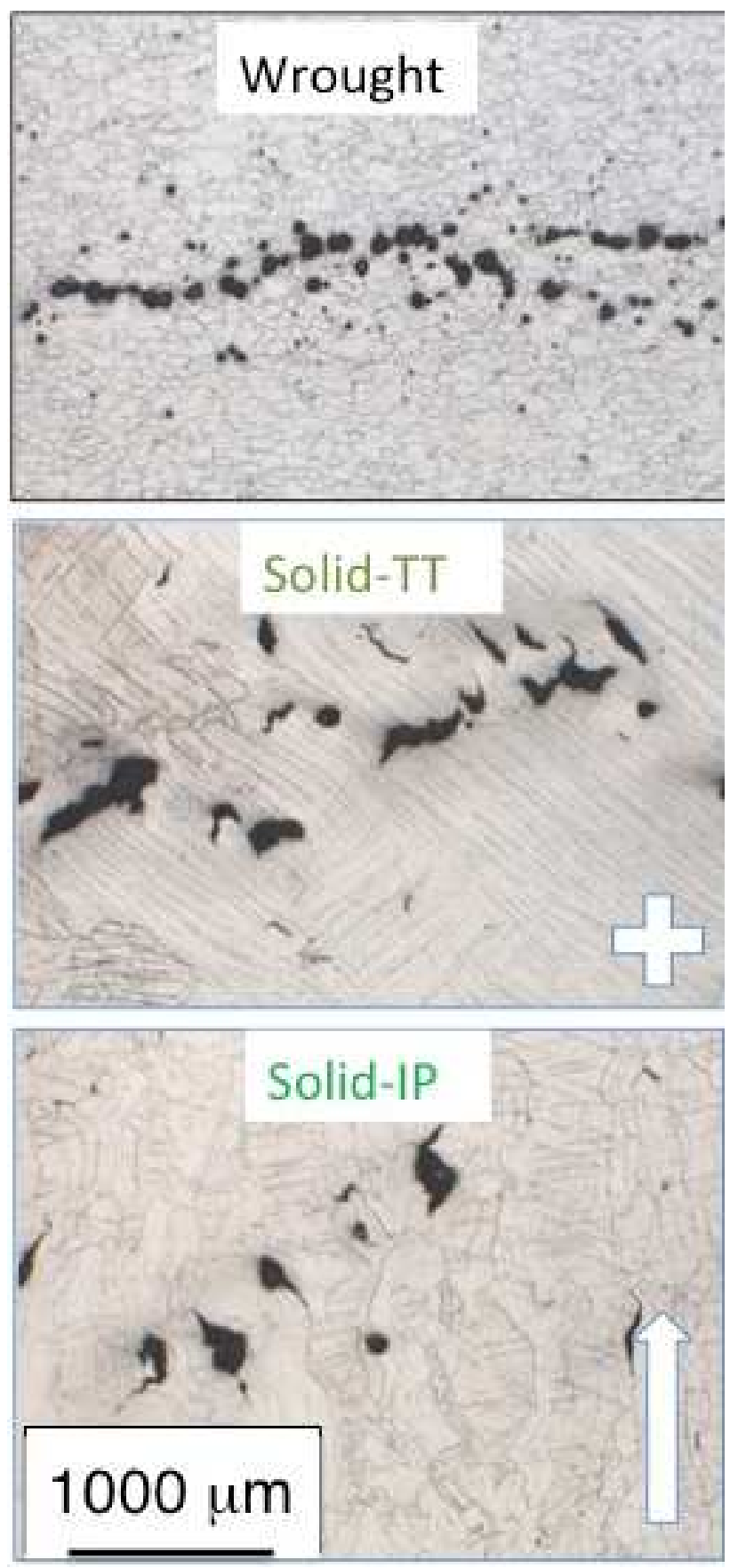

Fig. 7. Macroscopic optical metallography of incipiently spalled wrought $\mathrm{Ta}$ and the three AM-Ta materials. The arrow designates the AM growth direction while the "+" symbol is orthogonal to the AM growth direction (i.e., into the page).

In contrast, the three AM-Ta samples displayed a mixture of isolated void nucleation and growth interspersed with local shear localization and evidence of shear and interfacial delamination and/or cracking along the boundaries of the elongated grains. As the porous and solid TT samples displayed very similar damage evolution, only the solid-TT sample is presented in Figure 7. The linear features in both the solid-TT and solid-IP were identified to be planar dislocation slip lines lying along [110] planes. Higher magnification of these slip lines further reveals classic dislocation etch pits as seen in Figure 8. The observation of damage following the elongated grain boundaries suggests either reduced resistance to strain transmission across these boundaries, leading to shear stress concentration with increasing strain compatibility mismatch, or an inherent weakness of these boundaries perhaps due to impurity / solute concentration at these boundaries. Future research will concentrate on examining in detail both of these possibilities.

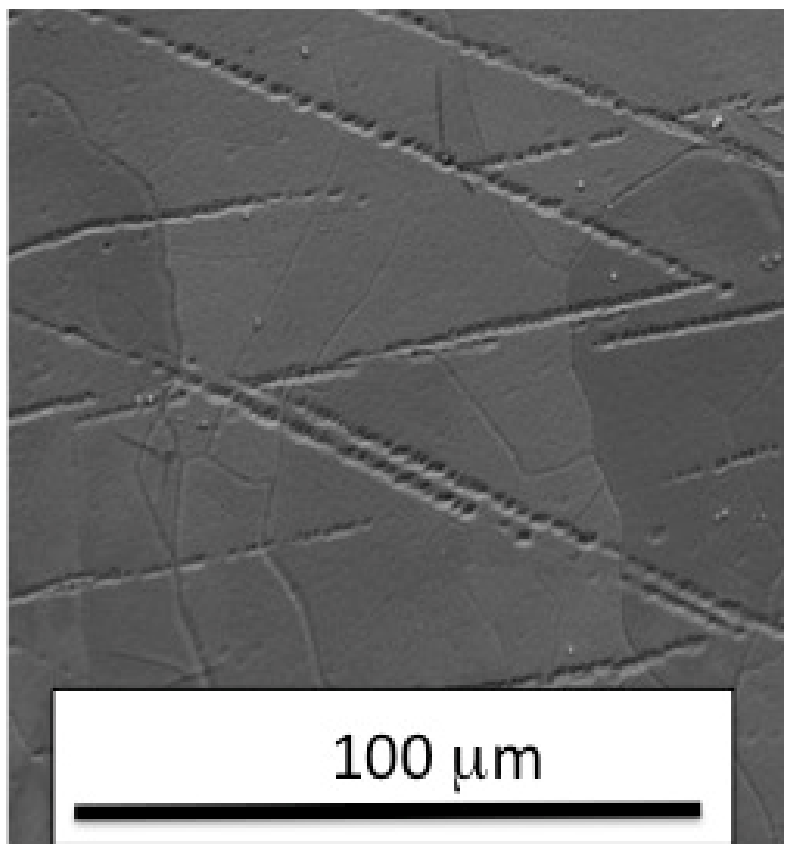

Fig. 8. Higher magnification metallography using scanning electron microscopy (SEM) illustrating that the linear dislocation slip lines display classic dislocation etch pits along the length of the dislocation lines.

\section{Summary}

The microstructure, quasi-static mechanical behavior, Hugoniot Elastic Limits (HEL), spall strength, and damage evolution of $\mathrm{AM} \mathrm{Ta}$ is demonstrated to be strongly dependent on the orientation of the AM build direction and substantially different than annealed wrought Ta. The HEL of the AM-Ta orthogonal to the elongated grain structure was found to be approximately a factor of two higher than the wrought Ta. The spall strength of the AM-Ta was less anisotropic than the grain structure. Overall, the dynamic damage evolution in the AM-Ta was found to be a mixture of ductile voids and shear damage along the grain structure in contrast to the wrought Ta which displayed classic void nucleation and growth with initiation principally at grain boundaries. Quantification of the dynamic deformation and damage behavior of AM materials is critical to the development of physically-based models of both the shock response of AM materials but also to the development of certification and qualification protocols for AM materials.

Los Alamos National Laboratory is operated by Los Alamos National Security, LLC, for the National Nuclear Security Administration of the U.S. Department of Energy under contract DE-AC52-06NA25396. This work was sponsored by the Laboratory Directed Research and Development (LDRD) program. 


\section{References}

1. W. E. Frazier, J. Matls. Eng. and Perf., 23, 1917 (2014)

2. W. J. Sames, F. A. List, S. Pannala, R. R. DeHoff, S. S. Babu, Int. Matls. Rev., 61, 315 (2016)

3. M. Seifi, A. Salem, J. Beuth, O. Harrysson, J. J. Lewandowski, JOM, 68, 747 (2016)

4. Y. Zhai, D. A. Lados, J. L. Lagoy, Journal of Metals, 66, 808 (2014)

5 B. R. Berger, Additive Manufacturing: A Game Changer for the Manufacturing Industry? (Berger STrategy Consultants, Munich, Germany, 2013)

6. G. T. Gray III, Annual Review of Materials Research, Vol 42, 42, 285 (2012)

7. R. Fadida, D. Rittel, A. Shirizly, J. Appl. Mech., 82, 041004 (2015)

8. D. R. Jones, S. J. Fensin, O. Dippo, R. A. Beal, V. Livescu, D. T. Martinez, C. P. Trujillo, J. N. Florando, M. Kumar, G. T. Gray III, J. Appl. Physics, 120, 135902 (2016)

9. G. T. Gray III, V. Livescu, P. A. Rigg, C. P. Trujillo, C. M. Cady, S. R. Chen, J. S. Carpenter, T. J. Lienert, S. J. Fensin, Acta Materialia, 138, 140 (2017)
10 S. I. Wright, D. P. Field, D. J. Dingley, In: Electron Backscatter Diffraction in Materials Science, edited by Schwartz AJ, Kumar M, Adams BL, Kluwer Academic / Plenum Publishers, New York, N.Y., p.141 (2000)

11.S. I. Wright, G. T. Gray III, A. D. Rollett, Metallurgical and Materials Transactions, 25A, 1025 (1994)

12.G. T. Gray III, In: ASM Handbook. Vol. 8: Mechanical Testing and Evaluation, edited by Kuhn H, Medlin D, ASM International, Materials Park, Ohio, p.530 (2000)

13. B. J. Jensen, D. B. Holtkamp, P. A. Rigg, D. H. Dolan, J. Appl. Phys., 101, 013523 (2007)

14. B. L. Adams, S. I. Wright, K. Kunze, Metallurgical Transactions A, 24A, 819 (1993)

15. P. S. Follansbee, Fundamentals of Strength (John Wiley \& Sons, Inc., Hoboken , N.J., 2014)

16. V. I. Romanchenko, G. V. Stepanov, Strength Mater., 9, 1110 (1977)

17. S. A. Novikov, I. I. Divnov, A. G. Ivanov, Phys. Metals Metallog., 21(4), 122 (1966)

18. G. T. Gray III, N. K. Bourne, K. S. Vecchio, J. C. F. Millett, Int. J. Fract., 163, 243 (2010) 